\title{
Effect of Streamwise Vortices on Characteristics of Jets*
}

\author{
Kuniaki TOYODA** and Riho HIRAMOTO**
}

\begin{abstract}
The interaction of streamwise and axisymmetric vortices in an axisymmetric jet is studied by flow visualization and velocity measurement. The jet is excited by azimuthal and axial perturbations to enhance streamwise and axisymmetric vortices. The three-dimensional views of the jet-boundary surface and streamwise vortices are constructed by applying the Taylor hypothesis to the jet cross-sectional images, and the interaction model of streamwise and axisymmetric vortices is proposed. The interaction of spanwise and streamwise vortices in a plane jet is also studied by velocity measurement. The experiment is carried out under conditions similar to those of the axisymmetric jet. The vortical structure is discussed on the basis of the three-dimensional views of phase-averaged vorticities. It is confirmed that the interacting vortical structure is similar to that in an axisymmetric jet. The entrainment mechanism is also discussed in relation to the vortical structure.
\end{abstract}

Key Words: Jet, Vortex, Flow Visualization, Digital Image Processing, Turbulent Mixing, Entrainment

\section{Introduction}

The use of streamwise vortices is noted as a useful technique for enhancing mixing in jets. In an axisymmetric jet, axisymmetric and streamwise vortices evolve and interact with each other. The interaction is threedimensionally complicated, and it is very difficult to detect the interacting structure experimentally by any other methods except flow visualization techniques. Several works have been reported to clarify the structure by flow visualization $^{(1)-(6)}$. Although the previous works give us useful information on the vortical structure, the interaction mechanism of axisymmetric and streamwise vortices is still unclear. In a plane jet, streamwise vortices evolve and interact with spanwise vortices. Although the evolutions of streamwise vortices are well known ${ }^{(7)}$, the details of the interaction are not understood.

In the present study, the details of axisymmetric/spanwise and streamwise vortices are clarified by the three-dimensional views of vortical structures, and the interaction mechanism of the vortices is discussed focusing on the deformation of vortices. The characteristics of the entrainment of axisymmetric and plane jets are also discussed in relation to the vortical structure. The results reveal the enhancement of entrainment by the interaction of

* Received 13th March, 2006 (No. 06-4064)

** Department of Mechanical Systems Engineering, Hokkaido Institute of Technology, 7-15-4-1 Maeda, Teine-ku, Sapporo 006-8585, Japan. E-mail: toyoda@ hit.ac.jp axisymmetric/spanwise and streamwise vortices.

\section{Axisymmetric Jet}

\subsection{Experimental apparatus and procedures}

In the experiments, flow visualization and mean velocity measurement were carried out using the water tunnel shown in Fig. 1. The water tunnel is of the overflow type, and the water head is kept at a constant level during the experiments. A vibrator is installed upstream to stabilize and enhance vortex evolution in the jet. A plastic plate connected with the vibrating rod shakes the water surface, and the longitudinal perturbation is transmitted to the jet.

The nozzle is shown in Fig. 2. The nozzle diameter is $46 \mathrm{~mm}$, and six vortex generators (VGs) are attached to the nozzle exit in order to enhance the streamwise vortices being generated in the initial mixing layer of the jet. The axisymmetric vortices were enhanced by the vibrator in Fig. 1. The excitation frequency is $f e=1.8 \mathrm{~Hz}$,



Fig. 1 Water tunnel 

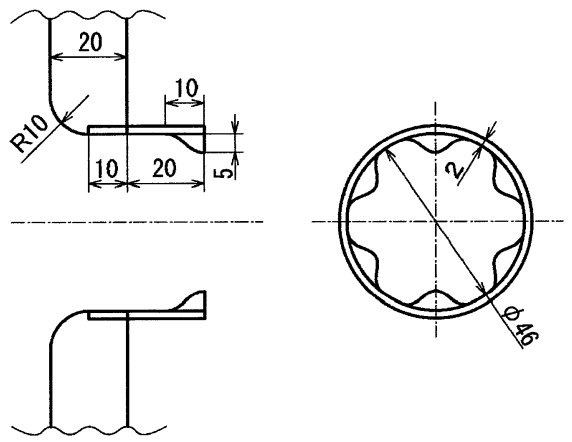

Fig. 2 Nozzle

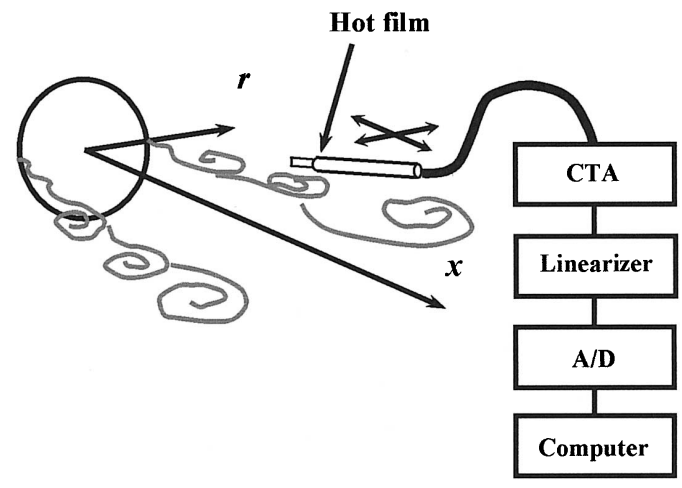

Fig. 3 Velocity measurement system

and the Strouhal number is $S t=f e D / U_{0}=0.69$. Under these conditions, large-scale axisymmetric vortex rings were evolved. The jet velocity at the center of the exit, $U_{0}$, is $0.12 \mathrm{~m} / \mathrm{s}$ and the Reynolds number is $3.8 \times 10^{3}$.

To visualize the jet, laser fluorescent dye (uranine) and a $2 \mathrm{~W}$ Argon-ion laser were used. The test section of the water tunnel was dyed with uranine at a low concentration prior to issuing clean water from the nozzle. The laser light was irradiated onto a thin sheet using a cylindrical lens to obtain the jet cross-sectional views. The cross-sectional views were recorded using a digital video camera fixed on the downstream side of the water tunnel. The views were recorded at the streamwise stations of $x / D=0 \sim 3.0$ ( $x$ is the streamwise distance from the jet exit and nondimensionalized by the nozzle diameter $D$ ).

The velocity measurement system is shown in Fig. 3 . Mean velocity profiles were measured using a hot-film probe over the flow field to discuss the entrainment of the jet. The traversing of the probe was controlled by a personal computer, and the velocity signals were recorded in the computer via an A/D converter. The data were analyzed with a data-processing computer system.

\subsection{Results and discussion}

The cross-sectional views of the jet at $x / D=0.75$ and 1.0 are shown in Fig. 4. The views reveal the evolution of streamwise vortices. By applying the Taylor hypothesis to the cross-sectional images recorded sequentially at each station, the three-dimensional views of jet-

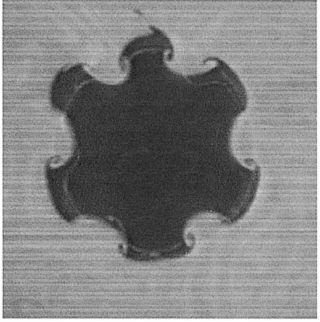

(a) $x / D=0.75$

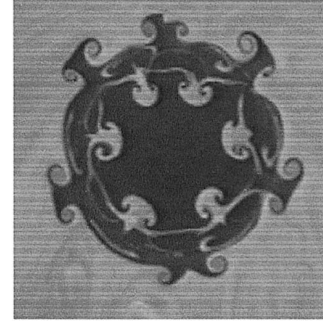

(b) $x / D=1.0$
Fig. 4 Jet cross-sectional views

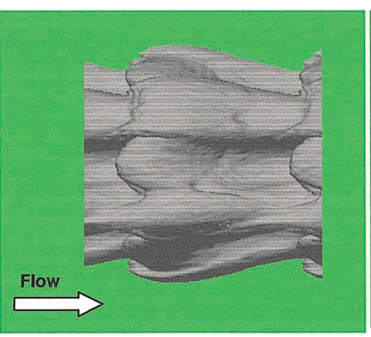

(a) Jet-boundary surface

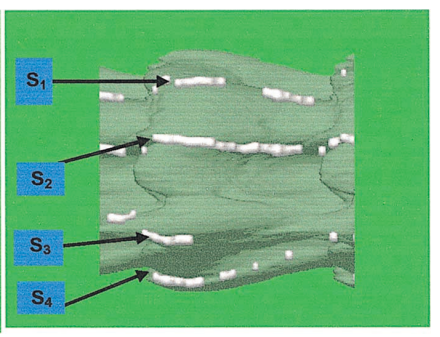

(b) Streamwise vortices
Fig. 5 Three-dimensional views at $x / D=0.75$

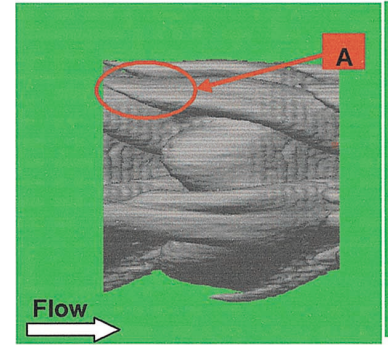

(a) Jet-boundary surface

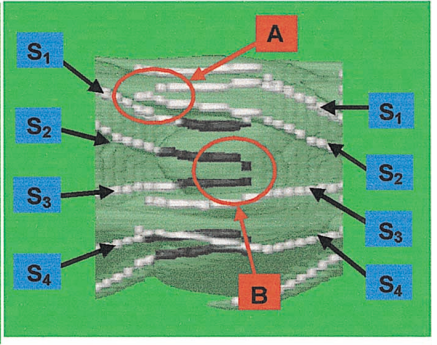

(b) Streamwise vortices
Fig. 6 Three-dimensional views at $x / D=1.0$

boundary surfaces are constructed as shown in Figs. 5 (a) and $6(a)$, where the convection velocity of the vortical structure is assumed constant. The jet-boundary surfaces are deformed by the effects of axisymmetric and streamwise vortices.

The streamwise vortices are detected from the crosssectional images and superposed on the jet-boundary surfaces as shown in Figs. 5 (b) and 6 (b), where the dark parts are located inside the axisymmetric vortex. The figures indicate how the streamwise vortices interact with the axisymmetric vortices. At the early stage of the evolution of streamwise vortices, the vortices are lined nearly parallel as shown in Fig. 5 (b). Farther downstream, the vortices are paired around the axisymmetric vortex as shown in Fig. 6(b): $S_{1}$ and $S_{2}$ are paired outside the axisymmetric vortex; $S_{2}$ and $S_{3}$ are paired inside the axisymmetric vortex.

Figures 5 and 6 lead to the interaction model in Fig. 7. At stage 1, the streamwise vortices are generated by VGs, and the axisymmetric vortex evolves via the 


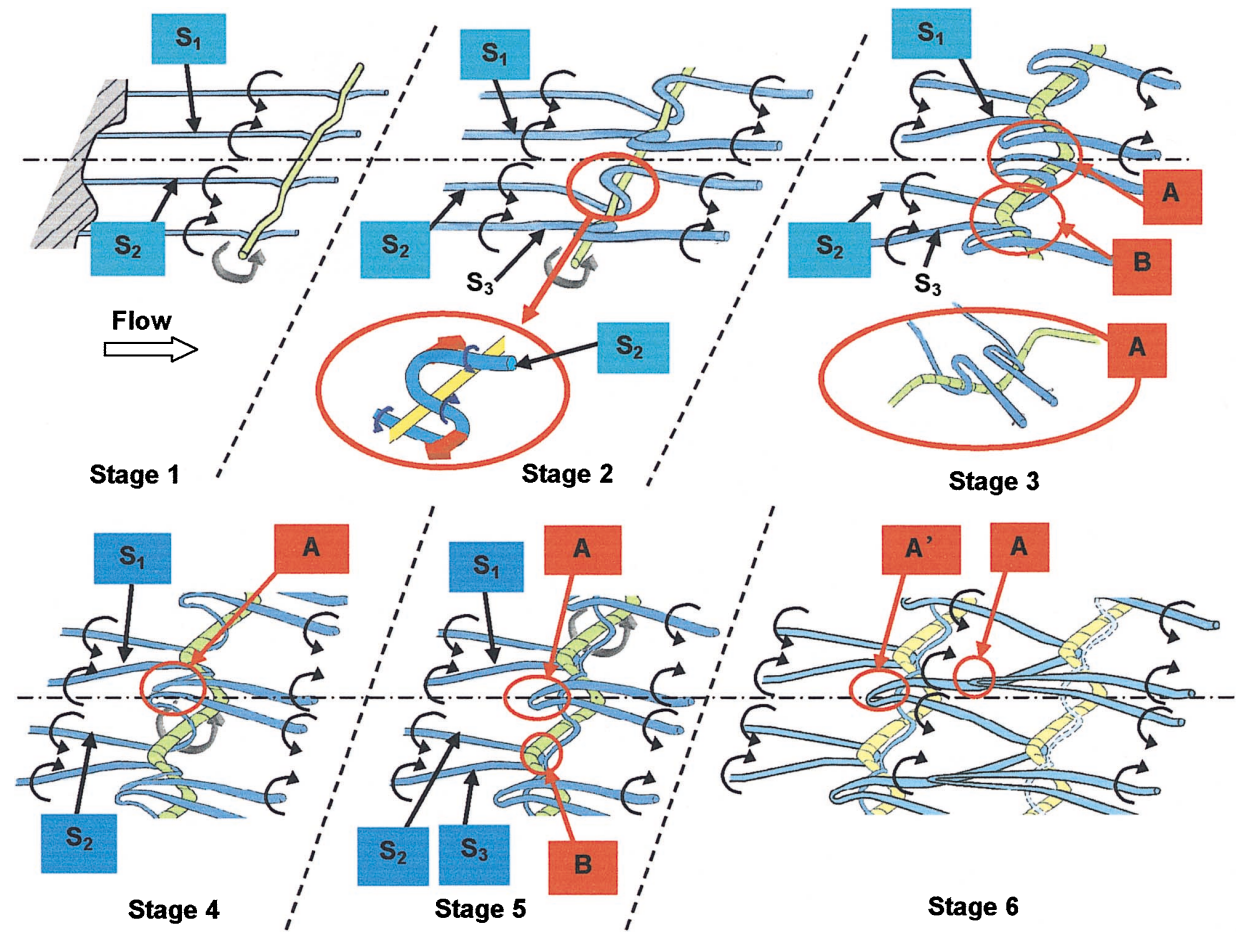

Fig. 7 Interaction model of axisymmetric and streamwise vortices

Kelvin-Helmholz instability. At stage 2, the streamwise vortices wind around the axisymmetric vortex. Outside the axisymmetric vortex, the streamwise vortex $S_{2}$ moves toward $S_{1}$ due to the self-induced velocity generated by the nonuniform curvature effect, and $S_{1}$ moves toward $S_{2}$ due to the opposite self-induced velocity. As a result, $S_{1}$ and $S_{2}$ move closer to each other (stage 3). Inside the axisymmetric vortex, $S_{2}$ and $S_{3}$ move closer to each other due to the self-induced velocity of each vortex. The paired streamwise vortices around the axisymmetric vortex contact each other from stage 4 to stage 5 and are connected via the cross-linking process ${ }^{(8)}$ (stage 5). The regions $A$ and $\mathrm{B}$ at stage 4 correspond to the regions $\mathrm{A}$ and $\mathrm{B}$ in Fig. 6 (b). The remainders of $S_{1}, S_{2}$ and $S_{3}$ are engulfed by the axisymmetric vortex as shown at stage 6 .

The entrainment ratio $\left(Q-Q_{0}\right) / Q_{0}$ of the jets are shown in Fig. 8, where $Q$ is the mass flow rate at a streamwise station and $Q_{0}$ is at the nozzle exit. It was calculated by integrating the mean velocity profiles in the radial direction on the assumption of axisymmetric velocity profiles. Figure 8 includes the results of unexcited and excited circular jets. The result of the excited jet with VG reveals that the enhancement of axisymmetric and streamwise vortices is very effective in increasing entrainment in the region of $x / D<2.0$. Farther downstream of $x / D=2.0$, the increasing rate of the entrainment ratio decreases probably due to the breakdown of vortices.

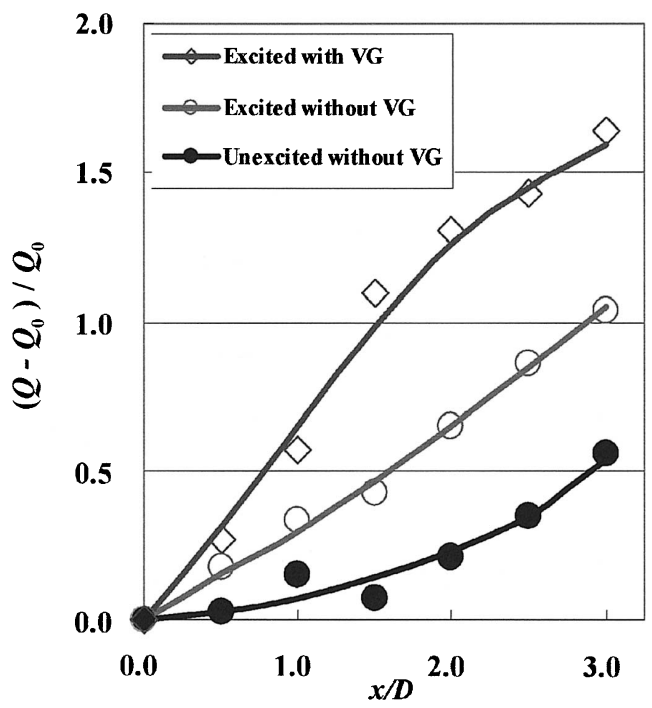

Fig. 8 Entrainment ratios

\section{Plane Jet}

\subsection{Experimental apparatus and procedures}

The relationship between the vortical structure and the entrainment in the initial region of a plane air jet with vortex generators at the nozzle exit was investigated by vorticity measurements. The nozzle and the coordinate system are shown in Fig. 9. The height $H$ and width $L$ of the nozzle exit are $50 \mathrm{~mm}$ and $400 \mathrm{~mm}$ respectively. The origin of the coordinate system is at the center of the nozzle exit. The vortex generators are attached to one side of the nozzle exit as shown in Fig. 10, since the initial re- 


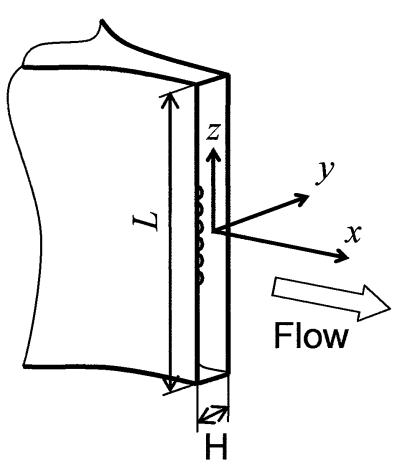

Fig. 9 Nozzle and coordinate system
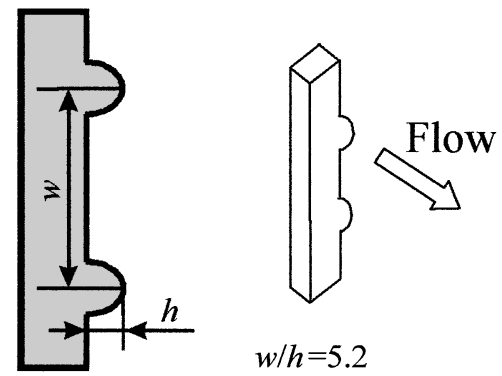

$w / h=5.2$

Fig. 10 Installation condition of VGs

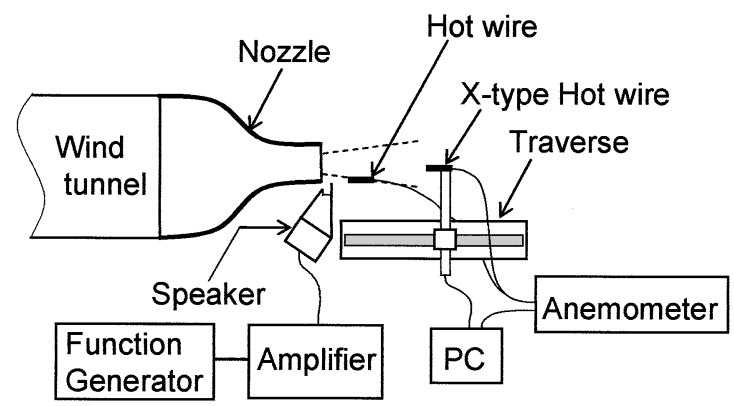

Fig. 11 Experimental apparatus

gion was focused. The height $h$ is $5 \mathrm{~mm}$ and the space $w$ is $26 \mathrm{~mm}$, and the ratio $w / h$ is 5.2 .

The experimental apparatus is shown in Fig. 11. The jet velocity $U_{0}$ at the center of the exit is $6.9 \mathrm{~m} / \mathrm{s}$ and the Reynolds number $\operatorname{Re}\left(=U_{0} H / v\right)$ is $2.4 \times 10^{4}$. The spanwise vortices were enhanced and stabilized with a speaker as shown in Fig. 11. The excitation frequency is $f e=66.3 \mathrm{~Hz}$, and the Strouhal number is $\mathrm{St}=\mathrm{feH} / U_{0}=0.48$. Under these conditions, the large-scale periodic vortices evolved, and they were detected by phase-average measurement. The reference signal for the phase-average processing was measured using a single normal hot-wire probe fixed near the nozzle exit. The phase-average processing was conducted at 36 phase angles of the reference signal from $\theta=0^{\circ}$ to $\theta=350^{\circ}$ with an increment of $10^{\circ}$. Three velocity components, in $x$-, $y$ - and $z$-directions, were measured with an X-type hot-wire probe controlled by a personal computer. The measurements were carried out twice at each measuring point to obtain the three velocity compo-

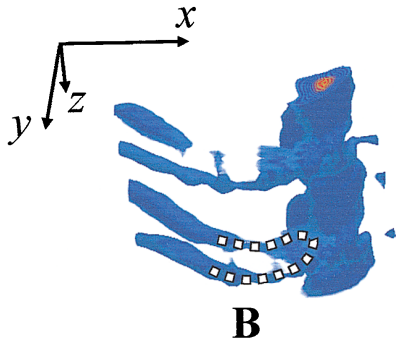

(a) View from jet center side

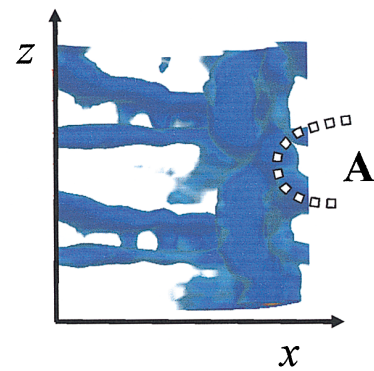

(b) View from outside
Fig. 12 Three-dimensional vortical structure $(x / H=0.75)$

nents by changing the rolling angle of the X-type hot-wire probe. The measuring period at each measuring point was $2 \mathrm{sec}$ at the sampling frequency of $5 \mathrm{kHz}$. The measurements in $y-z$ planes were conducted at $x / H=0.0,0.5$, $0.75,1.0,1.25$, and the measuring region is $y / H=0.0 \sim$ -1.3 and $z / H=0.0 \sim 1.6$ with the spatial interval of $1 \mathrm{~mm}$.

\section{2 Results and discussion}

3.2.1 Vortical structure The three-dimensional isosurfaces of phase-averaged absolute vorticity are shown in Fig. 12, which reveals the interacting vortical structure. The phase-averaged absolute vorticity is defined as follows.

$$
|<\omega>|=\left(<\omega_{\mathrm{x}}>^{2}+<\omega_{\mathrm{y}}>^{2}+<\omega_{\mathrm{z}}>^{2}\right)^{1 / 2}
$$

where $\left\langle\omega_{\mathrm{x}}\right\rangle,\left\langle\omega_{\mathrm{y}}\right\rangle$ and $\left\langle\omega_{\mathrm{z}}\right\rangle$ are the phase-average vorticities in the $x$-, $y$ - and $z$-directions, respectively. The isosurfaces are constructed by applying the Taylor hypothesis to the distributions of $|\langle\omega\rangle|$ in the $y-z$ plane at $x / H=0.75$. Figure 12 (a) shows the vortical structure viewed from the jet center side, and Fig. 12 (b) shows that viewed from the outside of the jet.

The hairpinlike structures indicated by 'A' and ' $\mathrm{B}$ ' in Fig. 12 are similar to the cross-linking structures at the stage 5 in Fig. 7. The result suggests that the interaction mechanism of streamwise and spanwise vortices in a plane jet is the same as that in an axisymmetric jet.

\subsubsection{Relationship between vortical structure and} entrainment The vortical structure and the distribution of entrainment ratio at $x / H=0.75$ are shown in Fig. 13 in the region of $y \leq 0.0$, where the flow field is affected by the vortex generators. In the figure, Line 1 shows the downstream of a vortex generator, and Line 2 shows the middle position between neighboring vortex generators. The entrainment rate is low in the braid region and high in the region where streamwise and spanwise vortices interact with each other. In the regions indicated by the circles in Fig. 13 (a), where the entrainment rate is higher, the streamwise vortices are reconnected to form hairpinlike vortices on the inner side of the spanwise vortex as mentioned in Fig. 12 (a).

To clarify the entrainment mechanism, the phaseaverage vorticity contours at the phase angle $\theta=70^{\circ}$ in 


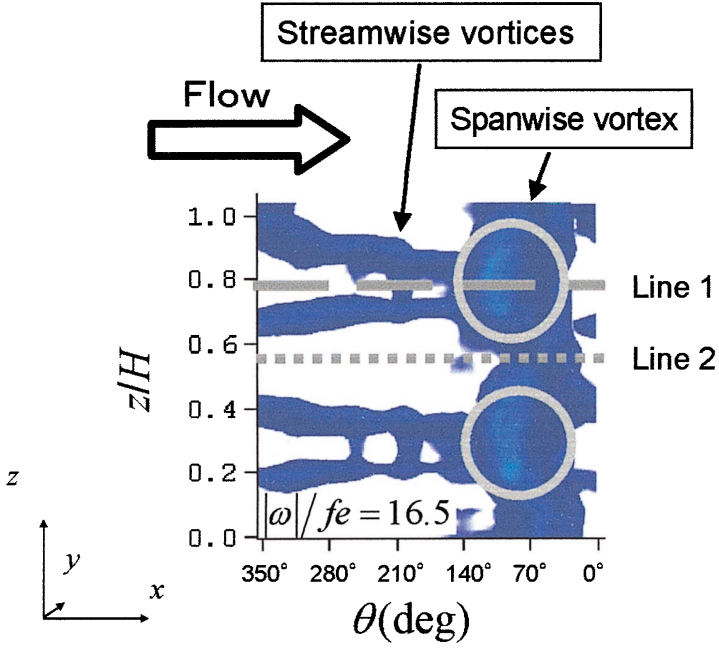

(a) Isosurface of $|<\omega>|$

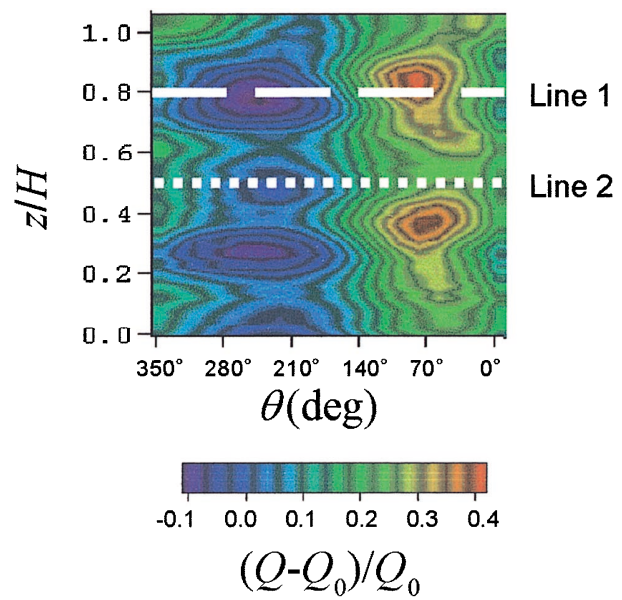

(b) Contours of entrainment ratio

Fig. 13 Vortical structure and entrainment rate $(x / H=0.75)$

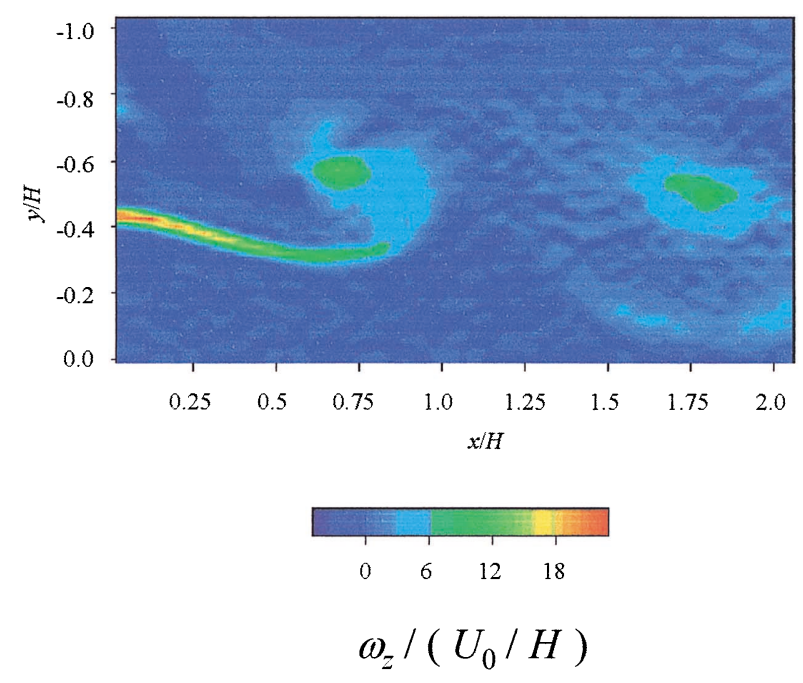

(a) $x-y$ plane along Line 1

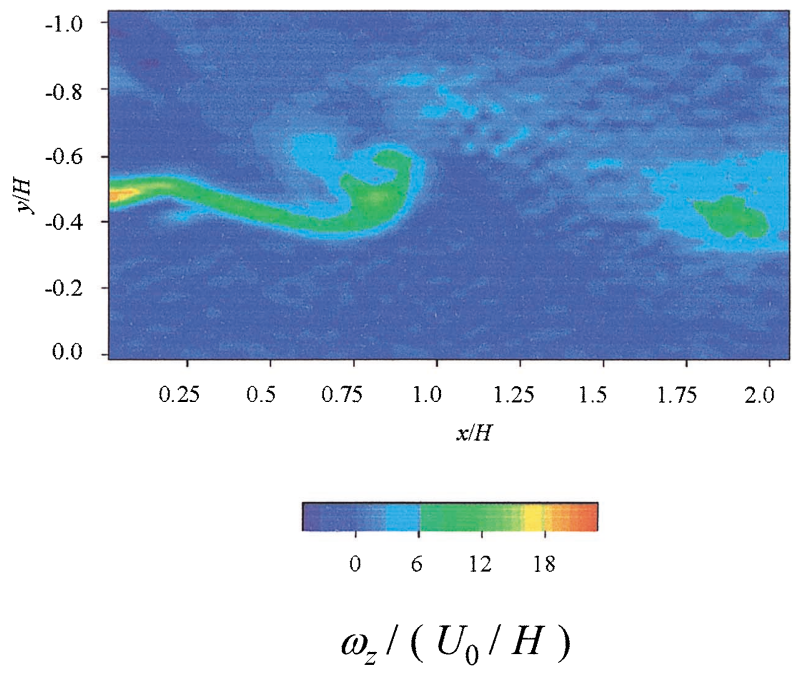

(b) $x-y$ plane along Line 2

Fig. 14 Contours of spanwise vorticity

the $x-y$ plane along Lines 1 and 2 are shown in Fig. 14. In the $x-y$ plane along Line 1 (Fig. 14 (a)), a large amount of outer fluid is engulfed into the jet, which leads to enhanced entrainment. The engulfment is due to the effect of velocity induced by the spanwise and hairpinlike vortices in Fig. 12 (a). In particular, the hairpinlike vortex is effective in engulfing the outer fluid. On the other hand, in the $x-y$ plane along Line 2, where the hairpinlike vortex outside the spanwise vortex in Fig. 12 (b) induces outer flow, the engulfment of outer fluid is weak as shown in Fig. 14 (b). These results reveal that the entrainment is closely related to the vortical structure.

\section{Conclusions}

The interaction mechanism of streamwise and axisymmetric vortices in an axisymmetric jet is clarified by analyzing visualization images. The streamwise vortices are paired, cut and reconnected around the axisymmetric vortex by the nonuniform curvature effect around the axisymmetric vortex. The entrainment is significantly increased by the enhancement of axisymmetric and streamwise vortices. The relationship between the vortical structure and the entrainment in a plane jet is also discussed with the details of phase-averaged vorticity. The crosslinking of streamwise vortices is confirmed in the initial region of a plane jet, and the mechanism of entrainment enhanced by the mutual effect of spanwise and streamwise vortices is clarified.

\section{Acknowledgments}

The authors are grateful to Mr. J. Akazawa and Mr. H. Mori for assistance in the experimental work. This study was partly supported by a Grant-in-Aid for Scientific Research [(C)(2) 13650191] from the Ministry of Education, 
Culture, Sports, Science and Technology of Japan.

\section{References}

( 1 ) Liepmann, D. and Gharib, M., The Roll of Streamwise Vorticity in the near Field Entrainment of Round Jets, J. Fluid Mech., Vol.245 (1992), pp.643-668.

( 2 ) Samimy, M., Zaman, K.B.M.Q. and Reeder, M.F., Effect of Tabs on the Flow and Noise Field of an Axisymmetric Jet, AIAA J., Vol.31, No.4 (1993), pp.609-619.

( 3 ) Reeder, M.F. and Samimy, M., The Evolution of a Jet with Vortex-Generating Tabs: Real-Time Visualization and Quantitative Measurement, J. Fluid Mech., Vol.311 (1996), pp.73-118.

( 4 ) Grinstein, F.F., Gutmark, E.J., Hanson-Parr, D.M. and Obeysekare, U., Streamwise and Spanwise Vortex Interaction in an Axisymmetric Jet. A Computational and
Experimental Study, Phys. Fluid, Vol.8, No.6 (1996), pp.1515-1524.

( 5 ) Toyoda, K., Muramatsu, Y. and Hiramoto, R., Visualization of the Vortical Structure of a Circular Jet Excited by Axial and Azimuthal Perturbations, J. Visualization, Vol.2, No.1 (1999), pp.17-24.

( 6 ) Toyoda, K. and Mori, H., Three-Dimensional Vortical Structure and Mixing Mechanism of a Circular Jet, J. Visualization, Vol.4, No.3 (2001), pp.239-244.

( 7 ) Lasheras, J.C. and Choi, H., Three-Dimensional Instability of a Plane Free Shear Layer, J. Fluid Mech., Vol.189 (1988), pp.53-86.

( 8 ) Melander, M.V. and Hussain, F., Cross-Linking of Two Antiparallel Vortex Tubes, Phys. Fluids A, Vol.1, No.4 (1989), pp.633-636. 